\title{
DETERMINAÇÃO DAS PROPRIEDADES DA TORTA OBTIDA NA FILTRAÇÃO DE FLUIDOS DE PERFURAÇÃO
}

\author{
L. A. SILVA ${ }^{1 *}$, FERRAZ, A. S. F. S¹, L. A. CALÇADA², C. M. SCHEID ${ }^{2}$ \\ ${ }^{1}$ Universidade Federal Rural do Rio de Janeiro, Programa de Pós-Graduação em \\ Engenharia Química \\ ${ }^{2}$ Universidade Federal Rural do Rio de Janeiro, Departamento de Engenharia Química \\ BR465, KM 7, Seropédica, Rio de Janeiro, CEP: 21941-030 \\ *e-mail: leandroazevedo.eq@gmail.com
}

\begin{abstract}
RESUMO
O Laboratório de Escoamento de Fluidos (LEF) da UFRRJ vem estudando o processo de filtração que acontece nas paredes do poço de petróleo durante a perfuração. Vários trabalhos foram realizados comparando o comportamento da filtração dinâmica e estática com fluidos de perfuração base água e base óleo e foram estimados parâmetros da torta como a porosidade, a permeabilidade, a espessura da torta etc. Neste trabalho, são apresentadas avaliações experimentais do efeito do tamanho de partículas nas curvas de filtração. As equações de filtração foram implementadas no software Matlab com o objetivo de simular o processo de filtração e invasão dos fluidos na rocha reservatório. Os resultados mostraram que o modelo e os parâmetros estimados em trabalhos anteriores possibilitaram a modelagem do processo.
\end{abstract}

\section{INTRODUÇÃO}

No processo de perfuração de poços de petróleo é necessário conhecer como se comporta a torta de filtração formada a fim de evitar invasões indesejadas do filtrado, que pode causar danos muitas vezes irreversíveis à rocha reservatório, tornando o poço inviável para a produção do óleo.

De acordo com Hwang, Chang e Chen (1996) o fenômeno da filtração ocorre basicamente, quando uma solução qualquer contendo sólidos em suspensão é pressurizada contra um meio poroso. Os componentes sólidos tendem a se depositar e se aderir na superfície do meio poroso exposta ao fluido, formando um filme. É essencial que o fluido tenha uma fração razoável de partículas com dimensões ligeiramente menores que as dimensões dos poros das rochas expostas, especificamente quando o assunto é perfusão de poço de petróleo. Quando existem partículas sólidas com dimensões adequadas, a obstrução dos poros é rápida e somente a fase líquida do fluido invade a rocha reservatória.

No processo de filtração ocorre a formação de uma torta, também conhecido reboco, esta torta pode ser externa ou interna à formação (ALSABA, NYGAARD, SAASEN e NES, 2014).

Pela teoria da filtração, de acordo com Carter (1957), duas fases distintas ocorrem durante o processo de filtração. $\mathrm{O}$ primeiro fenômeno é representado por uma fase inicial conhecida como 
spurt loss, Esta fase antecede a formação do reboco e, é representado pelo salto inicial do volume de filtrado antes da formação da torta. O segundo fenômeno é a consequente formação do reboco sobre a superfície do meio filtrante. O crescimento do reboco está diretamente associado à velocidade de filtração. Nesta fase, o fluxo de fluido da fratura para a formação é controlado pela resistência do reboco.

A filtração pode ser estática ou dinâmica. Na filtração estática quando a circulação do fluido de perfuração é interrompida, o reboco de baixa permeabilidade formado cresce continuamente sendo o responsável por controlar as taxas de filtração no poço. Portanto, na medida em que a espessura do reboco vai aumentando, as taxas de filtração vão diminuindo ao longo do tempo. Na filtração dinâmica quando há circulação do fluido, a espessura do reboco é função do equilíbrio dinâmico entre a taxa de deposição das partículas e a taxa de erosão da torta de filtração provocada pelo escoamento do fluido no poço. Sendo assim, quando esse equilíbrio é atingido o reboco adquire uma espessura constante $\mathrm{e}$ consequentemente as taxas de filtração no poço serão constantes também, ou seja, não haverá mais a erosão da torta e nem o crescimento da torta (LIU \& CIVAN, 1993).

O fluido de perfuração pode ser entendido de diversas formas na literatura, mas conceituando o fluido de uma maneira mais didática e ampla o mesmo pode ser compreendido como um líquido, líquido gaseificado ou um gás usado na perfuração de um poço de petróleo, sendo o mesmo bombeado até a broca e imergindo pelo anular.

O fluido de perfuração também é usado para tentar impedir ou minimizar a invasão na rocha reservatório. A indústria tem proposto algumas ideias, a maioria delas baseada na adição de agentes tamponadores, para a formulação da composição do fluido de perfuração. Tais agentes bloqueariam os poros da rocha perto da parede do poço, restringindo, consequentemente, a invasão do filtrado do fluido na rocha (WALDMANN, 2005).

A formação da torta na parede do reservatório pode causar danos irreversíveis ao poço. Didaticamente pode-se dizer que este reboco formado na parede do poço do reservatório leva ao entupimento dos veios aonde o óleo irá lixiviar durante a extração do óleo, por isso é fundamental o estudo do crescimento e do comportamento deste material depositado nessa região durante o escoamento axial entre a coluna de perfuração e o anular.

Os fluidos de perfuração são muito importantes durante as operações de perfuração, pois desempenham uma série de funções essenciais. Este desempenho é diretamente dependente das propriedades reológicas, de filtração e lubricidade (viscosidades, consistência de gel, controle de filtrado, formação reboco e coeficiente de lubricidade) dos fluidos de perfuração (THOMAS, 2001).

No estudo da filtração de fluidos de perfuração alguns trabalhos já foram desenvolvidos na LEF/UFRRJ em parceria com o CENPES e Petrobras. Os principais autores são: Araújo (2010), Martins (2013) e Calabrez (2013) e Ferraz (2014). Neste artigo serão abordados os experimentos de Ferraz (2014) para avaliação do efeito da granulometria de carbonato de cálcio nas curvas de filtração utilizando as principais equações que modelam 0 processo de filtração. Os dados experimentais serão modelados a partir de equações da literatura com o auxílio 
do software Matlab sendo o principal objetivo avaliar o desempenho destas equações na simulação do processo de filtração e invasão dos fluidos na rocha reservatório.

\section{EQUACIONAMENTO}

A avaliação dos parâmetros do processo de filtração tais como: porosidade, permeabilidade, compressibilidade, espessura, resistência ao cisalhamento e o fator de fricção da torta são relevantes para evitar invasões indesejadas do filtrado, que podem causar danos muitas vezes irreversíveis à rocha reservatório, tornando o poço inviável para a produção do óleo.

Dewan e Chenevert (2001) estudaram uma teoria que descreve o crescimento da torta e invasão de filtrado. A formulação a seguir, representa o escoamento de um fluido através de um meio poroso saturado, provocado por um diferencial de pressão imposto, a filtração estática. $\mathrm{O}$ fluido por conter sólidos, ao escoar no meio poroso forma uma torta sobre sua superfície, como mostra a Figura 1.

A formação do reboco nas paredes do poço é um fator importante do ponto de vista da perda de filtrado e de controle de danos à formação. Uma torta com baixa permeabilidade reduz a invasão de fluido na formação. Este cenário faz com que a taxa de filtração se torne independente da pressão de perfuração em condição overbalanced. Esse fato ocorre porque a permeabilidade da torta diminui com o aumento da pressão (JIAO \& SHARMA, 1993).
Figura 1 - Esquema de filtração linear com formação de torta

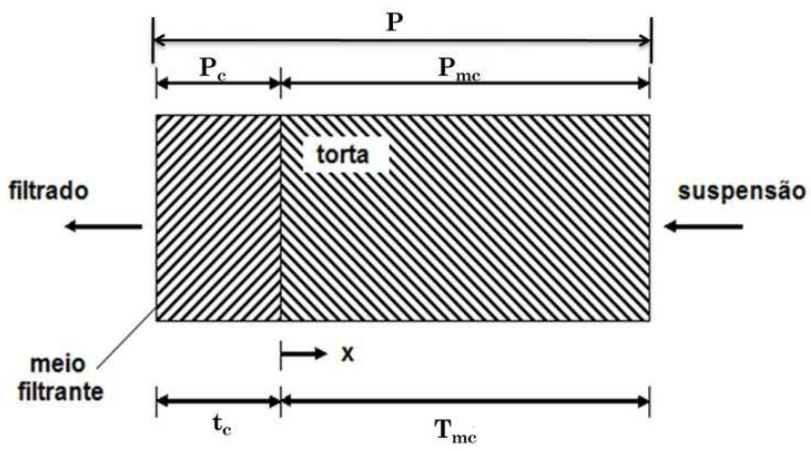

Fonte: Dewan \& Chenevert (2001).

Onde, $t_{c}$ é espessura do meio filtrante, $\mathrm{T}_{\mathrm{mc}}$ a espessura da torta de filtração, $\mathrm{P}_{\mathrm{mc}}$ a pressão através da torta, $\mathrm{P}$ a pressão aplicada.

A taxa de filtrado em meios porosos é comumente descrita pela Equação de Darcy (1856), Equação 1.

$$
q(t)=\frac{\left(P-P_{m c}\right) \cdot \mathrm{K}_{c}}{14700 \cdot \mu \cdot t_{c}}
$$

Sendo, $q(t)$ a taxa de filtração, $P_{m c}$ (psi) a pressão através da torta, $k_{c}(\mathrm{mD})$ a permeabilidade do meio filtrante, $\mu$ $(\mathrm{cP})$ a viscosidade do filtrado e $t_{c}(\mathrm{~cm})$ a espessura do meio filtrante.

Da mesma forma, a Equação de Darcy é aplicada ao cálculo da queda de pressão através da torta pela Equação 2.

$$
P_{m c}(t)=14700 \frac{q(t) \cdot T_{m c}(t) \cdot \mu}{k_{m c}(t)}
$$

Sendo, $k_{m c}$ a permeabilidade da torta, $\mu$ (cP) a viscosidade do filtrado, $q(t)$ a taxa de filtração e $T_{m c}(\mathrm{~cm})$ a espessura da torta de filtração. A taxa de filtrado também pode ser escrita de acordo com a Equação 3, onde A é a área de filtração, $\mathrm{A}\left(\mathrm{cm}^{2}\right)$. 


$$
q(t)=\frac{d v}{A \cdot d t}
$$

Igualando as equações 2 e 3 obtém se a Equação 4 que pode ser resolvida integrando a região onde se procura a solução e aplicando conceitos. Uma das condições de contorno do problema é que na Equação 4 inicialmente $(\mathrm{t}=0)$ não há volume de filtrado.

$$
\frac{d v}{d t}=14700 \frac{\mathrm{K}_{m c}(t) \cdot A \cdot P_{m c}(t)}{\mu \cdot T_{m c}(t)}
$$

De acordo com Tiller e Cooper (1962) é possível correlacionar a permeabilidade da torta compressível com uma variação de pressão aplicada sobre a mesma. Se o valor do índice de compressibilidade ( $\beta$ ) for zero, tem-se uma torta incompressível. Se $(\beta)$ atingir a unidade tem-se uma torta tão compressível que a sua permeabilidade é inversamente proporcional ao diferencial de pressão através dela. A Equação 5 de Dewan \& Chenevert (2001) estima esse comportamento.

$$
\mathrm{K}_{m c}(t)=\frac{\mathrm{K}_{m c} 0}{P_{m c}^{\beta}(t)}
$$

Onde, $k_{m c} 0$ é a permeabilidade de referência definida para uma pressão diferencial de 14,7 psi e $\beta$ é um expoente de compressibilidade.

Quando um aumento de pressão é aplicado contra o fluido que escoa pelo meio filtrante a mudança de pressão gera uma queda brusca no valor de slowness.

Fazendo o balanço de massa de sólidos na torta e utilizando a Equação 6 pode-se estimar a espessura da torta.
$\varphi=\frac{V_{\text {total }}-V_{\text {vazios }}}{V_{\text {total }}}$

Onde $\varphi$ é a porosidade, $V_{\text {total }}$ é o volume total da torta e $V_{\text {vazios }}$ o volume de vazios.

No software Matlab foram implementadas as equações citadas no artigo respeitando as condições dos experimentos. Foi utilizada a permeabilidade e a porosidade experimental. Nessas condições foi possível modelar as curvas de Slowness e a curva de volume de filtrado versus tempo além de calcular o volume de filtrado final e a espessura da torta.

\section{MATERIAIS E MÉTODOS}

Para realização deste estudo três diferentes faixas de distribuição granulométrica de carbonatos de cálcio foram adicionadas a fluidos de perfuração base água para avaliar o comportamento da curva de volume de filtrado e estimar os parâmetros da filtração.

\subsection{Caracterização de Tamanho das Partículas}

As partículas de carbonato de cálcio utilizadas foram separadas no agitador eletromagnético de peneiras nas faixas de 0-53 e 53-106 $\mu \mathrm{m}$.

A caracterização de tamanho das partículas foi realizada no analisador de partículas Malvern Mastersizer. A Figura 2 apresenta o resultado da caracterização para o carbonato de cálcio. 
Figura 2 - Distribuição granulométrica

do carbonato de cálcio.

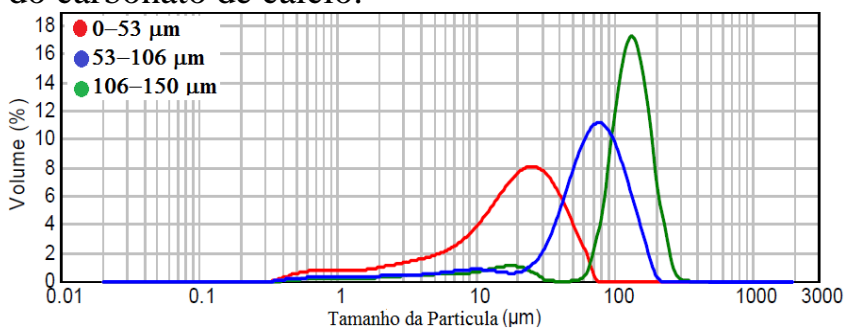

Fonte: Autor

\subsection{Célula de Filtração}

Para a realização dos experimentos, utilizou-se uma célula de filtração de alta pressão e alta temperatura, conhecida como HTHP (High Temperature, High Pressure). A célula produzida pela OFI, Testing Equipments (OFITE - 11302 Steeplecrest Dr. - Houston, TX 77065) e modificada pelo LEF- UFRRJ (Laboratório de Escoamento de fluidos Giulio Massarani - Universidade Federal Rural do Rio de Janeiro) é ilustrada na Figura 3.

O tempo total do experimento foi de no máximo 1 hora e seguiu as seguintes etapas: $O$ experimento foi iniciado com uma corrida de 30 minutos, aplicou-se uma pressão no sistema de 500 psi e cisalhamento zero. Nos 30 minutos finais aplicou-se uma pressão de 1000 psi e cisalhamento zero.
Figura 3 - Célula HTHP acoplada ao sistema de aquisição de dados

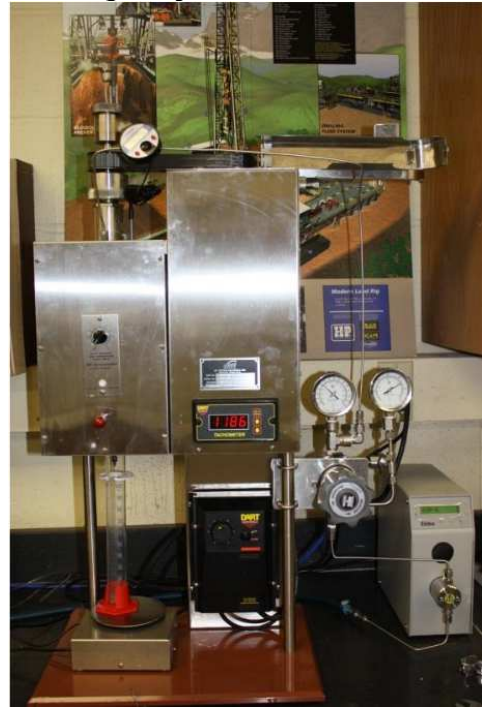

Fonte: Autor.

\section{RESULTADOS E DISCUSSÃO}

Os fluidos em solução polimérica, CMC à base de água, contendo carbonato de cálcio, permitiram avaliar o efeito das partículas que apresentam interação com o fluido, conforme a Tabela 1.

Tabela 1 - Dados de filtração de partículas de $\mathrm{CaCO}_{3}$ em solução de $\mathrm{CMC}$.

\begin{tabular}{ccc}
\hline Fluido & Componentes & $\begin{array}{c}\text { Volume de } \\
\text { filtrado (ml) }\end{array}$ \\
\hline Fluido 10 & $\begin{array}{c}\text { Carbonato de } \\
\text { cálcio (0-53 } \\
\mu \mathrm{m}) \text { e CMC }\end{array}$ & 12,71 \\
Fluido 11 & $\begin{array}{c}\text { Carbonato de } \\
\text { cálcio (53-106 } \\
\mu \mathrm{m}) \text { e CMC }\end{array}$ & 14,8 \\
& & \\
\hline
\end{tabular}

Fonte: Ferraz (2014).

A taxa de filtração foi calculada a partir das curvas de volume de filtrado versus tempo. A Figura 4 apresenta as curvas de filtração dos Fluidos 10, 11 e 12 e a Figura 5 apresenta as curvas de 
filtração e curva de Slowness dos experimentos 1.1,1.2 e 1,3 para o Fluido 10 (treplica).

Figura 4 - Volume de filtrado versus tempo para os Fluidos 10, 11 e 12 (CMC).

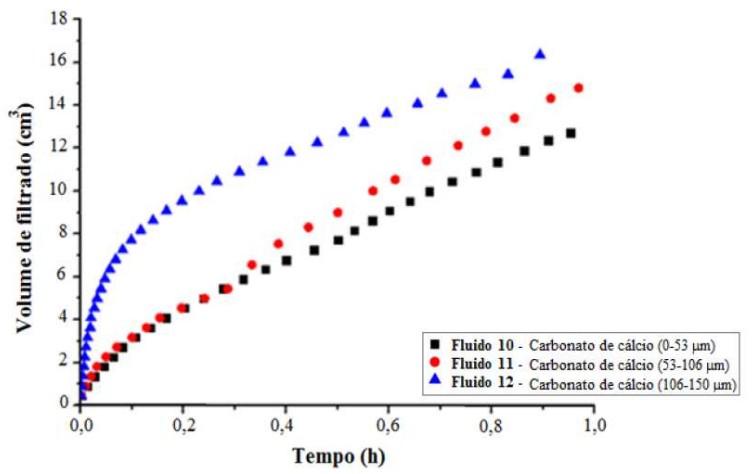

Fonte: Autor

Figura 5 - Curvas de slowness versus tempo - (Triplicata para o Fluido 10).

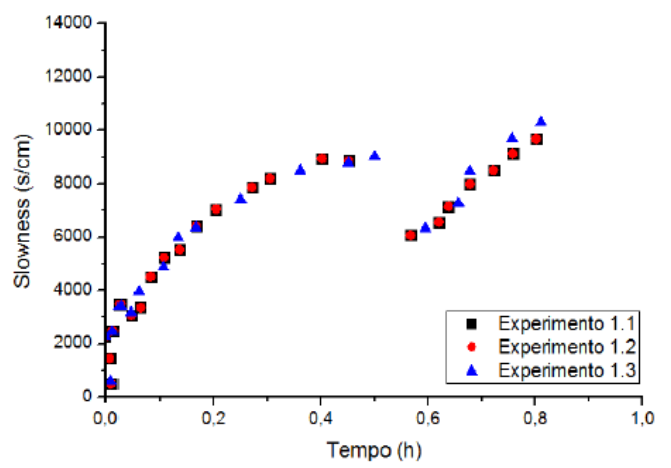

Fonte: Autor

Nas soluções de CMC o maior volume de filtrado foi obtido para o maior tamanho de partículas de $\mathrm{CaCO}_{3}$. Este resultado já era esperado em função da dificuldade de empacotamento das partículas de maior tamanho.

As Figuras 6 e 7 apresentam uma comparação entre curva experimental e a curva obtida utilizando a modelagem proposta neste artigo.

O coeficiente de determinação utilizado, também chamado de $\mathrm{R}^{2}$, é uma medida de ajustamento de um modelo estatístico linear generalizado, como a regressão linear, em relação aos valores observados. $\mathrm{O} \mathrm{R}^{2}$ indica em percentagem $\mathrm{O}$ quanto à equação consegue modelar os valores experimentais.

É possível notar visualmente nas Figuras 6 e 7 que as equações propostas modelaram bem a curva de filtração, além da avaliação qualitativa do coeficiente de determinação disposto nas figuras.

Figura 6 - Modelagem da curva de volume de filtrado versus tempo do Fluido 10, experimento 1.1.

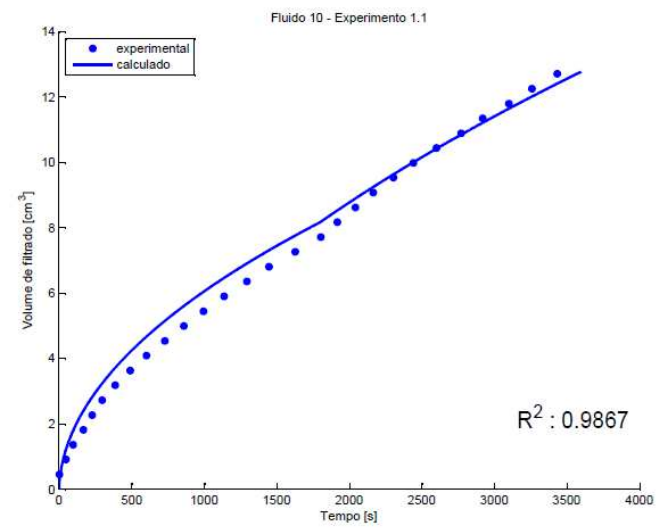

Fonte: Autor

Figura 7 - Modelagem da curva de volume de filtrado versus tempo do Fluido 11, experimento 1.1.

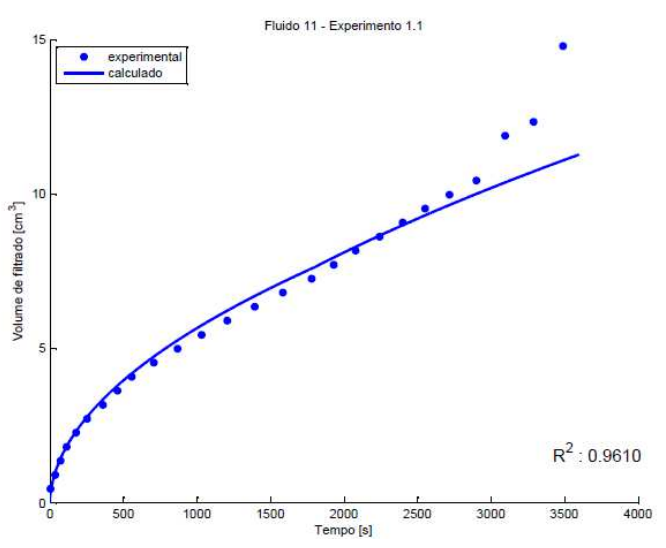

Fonte: Autor 
As curvas de Slowness do Fluido 10 e 11 estão dispostas nas Figuras 8 e 9.

Figura 8 - Modelagem da curva de Slowness do Fluido 10, experimento 1.1 .

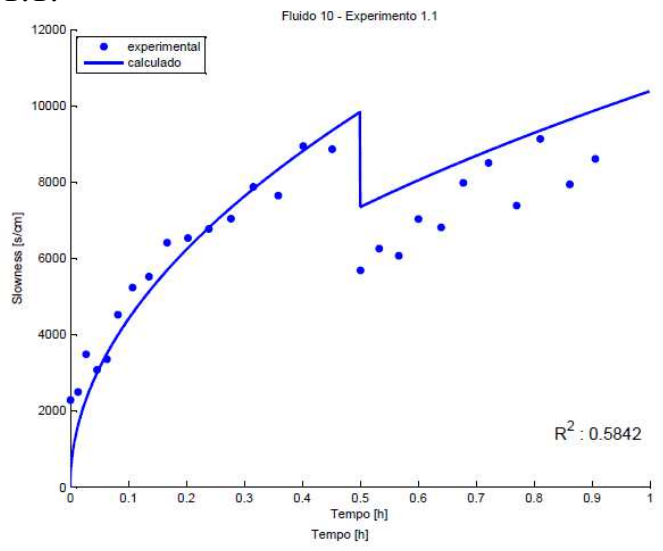

Fonte: Autor

Figura 9 - Modelagem da curva de Slowness do Fluido 11, experimento 1.1 .

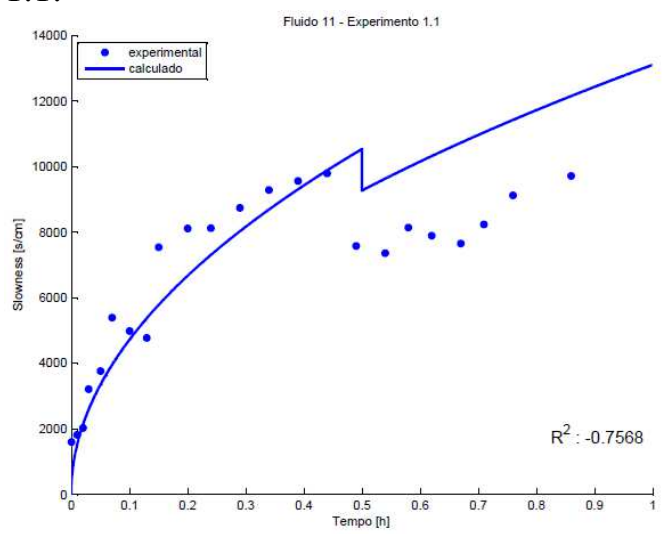

Fonte: Autor

As curvas de Slowness apresentam boa performance durante a primeira fase do experimento, quando a pressão aplicada ao sistema é de 500 psi. Tal fato pode ser atrelado ao comportamento da torta quando é comprimida pelo aumento de pressão. Visualmente pode se notar que para uma faixa granulométrica menor (Fluido 10) a equação teve um comportamento melhor do que para uma faixa granulométrica maior (Fluido
11). Além da analise visual é possível avaliar também os coeficientes de determinação dispostos nas Figuras 8 e 9.

A Tabela 2 apresenta a comparação entre a espessura da torta calculada e experimental, além do volume de filtrado e os parâmetros do Slowness.

Tabela 2 - Comparação entre parâmetros calculados e dados experimentais.

Fluido $10 \quad$ Fluido 11

Espessura calculada

(cm)

Espessura

experimental $(\mathrm{cm})$

0,80

Volume de filtrado calculado $\left(\mathrm{cm}^{3}\right)$

Volume de filtrado experimental $\left(\mathrm{cm}^{3}\right)$

12,71 14,80

\section{CONCLUSÃO}

A modelagem das curvas foi feita a partir dos dados experimentais de permeabilidade e porosidade do experimento 1.1 do fluido 10 e 11 .

Como já esperado as soluções de CMC com maior volume de filtrado foram obtidos para o maior tamanho de partículas de $\mathrm{CaCO}_{3}$. Este fenômeno ocorre devido a maior dificuldade de empacotamento das partículas de maior tamanho. Quanto maior o tamanho de partícula mais espaços vazios haverá na torta formada gerando menor perda de carga quando comparada a torta com composição de partículas menores.

A típica curva de volume de filtrado versus tempo para o processo de filtração é mostrada nas Figuras 6 e 7. 
A Tabela 2 lista o volume de filtrado experimental e calculado além da espessura da torta experimental. $O$ desvio entre os dados experimentais e os calculados pode estar atribuído à permeabilidade na torta de filtração. Quanto maiores as partículas contidas na torta maior será a dificuldade de organização das partículas, possibilitando ao sistema gerar comportamentos desconhecidos que as equações não podem modelar. Além disso pode se atribuir os desvios dos dados experimentais com a dificuldade de modelar os dados experimentais para a pressa de 1000 psi uma vez que o volume de filtrado é o ultimo ponto da curva de filtração, quando o tempo é 30 minutos.

A linha de pesquisa do trabalho pode levar ao entendimento da necessidade da otimização das equações de filtração com a adição de parâmetros que modelem o comportamento do experimento quando submetido à variação de pressão para as condições experimentais utilizadas neste caso. De acordo com o coeficiente de determinação as curvas de filtração apresentaram boa simulação com os dados experimentais. Pode ser observado visualmente que as Curvas de Slowness apresentaram bom desempenho na primeira metade do experimento, na pressão de 500 psi. Quando a pressão sobe para 1000 psi, as curvas não modelam bem $o$ experimento e quanto maior a granulometria maior ainda será a defasagem quando comparado a curva gerada pelo modelo com os dados experimentais.

Neste trabalho foram apresentadas avaliações do tamanho de partículas nas curvas de filtração. Ainda foi apresentado curvas a partir de parâmetros experimentais. As equações de filtração foram implementadas com o objetivo de simular o processo de filtração e invasão dos fluidos na rocha reservatório. Os resultados mostraram que o modelo e os parâmetros estimados em trabalhos anteriores possibilitaram a modelagem do processo, em uma determinada região da curva, início do experimento. No fim do experimento é necessário prever outros parâmetros das equações a fim de mostrar uma modelagem mais próxima da realidade. Da mesma forma para o cálculo de espessura da torta e volume final de filtrado.

\section{REFERÊNCIAS}

ALSABA, M., NYGAARD, R., SAASEN, A., and NES, O., 2014. Lost Circulation Materials Capability of Sealing Wide Fractures. SPE 170285, SPE Deepwater Drilling and Completions Conference, Galveston, USA, 10-11 September 2014.

ARAÚJO, C.A.O., "Estudo da Filtração Cruzada em Geometria Cilíndrica". Dissertação de Mestrado, PPGEQ/UFRRJ, Seropédica, Rio de Janeiro, Brasil, 2010.

CALABREZ, N.D; Filtração e Invasão de Fluidos de Perfuração: Estudo Comparativo, Caracterização da Torta e Modelagem. Tese de Mestrado Universidade Federal Rural do Rio de Janeiro, 2013- pp. 87.

CARTER, W.D.; "Disconformity between Lower and Upper Cretaceous in western Colorado and eastern Utah": Geological Society of America Bulletin, v 68, p. 307- 314, 1957. 
DEWAN, J. T. and CHENEVERT, M. E. A model for filtration of water-base mud during drilling: determination of mud cake parameters. Petrophysics, vol. 42, no. 3, pp. 237-250, 2001.

FERRAZ, A. S. F. S., Efeito da distribuição granulométrica de partículas sólidas e de polímeros aniônicos na formação da torta de filtração e no volume de filtrado 2014 . 108 p. Dissertação (Mestrado em Engenharia Química, Tecnologia Química). Instituto de Tecnologia, Departamento de Engenharia Química, Universidade Federal Rural do Rio de Janeiro, Seropédica, RJ, 2014.

HWANG, S.J.; CHANG, D.J.; CHEN, C. H. "Steady state permeate flux for particle cross-flow filtration". The Chemical Engineering Journal, 61, p.171-178, doi.org/10.1016/0376-7388 (94)00180-7, 1996.

JIAO, D. and SHARMA, M. M. Mechanism of buildup in cross flow filtration of colloidal suspensions. Journal of colloid and interface science, vol. 162, pp. 454-462, 1993.

LIU, X., CIVAN, F., 1993, “A Multiphase Mud fluid Infiltration and filter Cake formation model", In: SPE International Symposium on Oilfield Chemistry, SPE 25215, New Orleans, EUA, 2-5 March, 1993.

THOMAS, J. E.; "Fundamentos de Engenharia de Petróleo"; $2^{\circ}$ Edição; Editora Interciência, Rio de Janeiro, 2001.

TILLER, F.M., COOPER, H. The role of porosity in filtration: Part $\mathrm{V}$ Porosity variation in filter cakes.
AIChE Journal, vol. 8, pp. 445-449, 1962.

WALDMANN, A. T. A. "Mecanismos que governam a efetividade de agentes obturantes no controle da invasão de fluidos de perfuração na rocha reservatório de petróleo". Dissertação (Mestrado) - Rio de Janeiro: Pontifícia Universidade Católica do Rio de Janeiro, 2005.

\section{AGRADECIMENTOS}

Agradeço o apoio financeiro do CENPES/Petrobras e a CAPES. Agradeço o apoio do PPGEQ/UFRRJ. 